\title{
Les expérimentations graphiques de Julie Delporte
}

\section{Sylvain RHEAULT, Université de Regina}

Pourquoi ne pas profiter de ce format assez libre, un peu différent de l'article traditionnel, pour faire de «l'expérimentation »? Par ce moyen, je pourrai illustrer ma lecture des œuvres de Julie Delporte que je me prépare à analyser. Au lieu d'encadrer mon texte dans une structure traditionnelle, je laisserai surgir les idées telles qu'elles se présentent à l'esprit, aussi fragmentaires soient-elles. S'il fallait me référer à un modèle, j'opterais pour celui adopté par François Paré dans son bel essai sur Les Littérature de l'exiguïté. Tant qu'à y être, pourquoi ne pas risquer, sans filet, certaines hypothèses quant à l'expérimentation en BD ? C'est-à-dire discuter sans nécessairement compter sur l'autorité des prédécesseurs. C'est-à-dire risquer de se tromper pour aider ceux qui suivent à trouver la vérité.

Je ne me dis jamais : «Bon, je vais sortir de la convention, je vais faire autre chose ", mais cela me vient naturellement. Je préfère les artistes qui expérimentent, quitte à ce que ce soit brouillon, plutôt que les gens qui suivent des modèles très établis. De la bande dessinée en peinture, aujourd'hui ce n'est plus si étonnant, mais c'est quand même assez récent, et c'est encore un peu de l'expérimentation suivant les cas. (Julie Delporte, dernier commentaire de l'entrevue du 31 juillet 2013)

Puisqu'il est question d'expérimentation, il faudrait réfléchir au mot. S'agit-il d'aller où les autres avant soi n'ont jamais mis le pied ? S'agit-il de tourner à droite quand tout le monde tourne à gauche ? Faut-il renier ses devanciers et tout réinventer en faisant table rase de la tradition? Faut-il, pour le ou la bédéiste, toujours favoriser son propre choix au détriment de ce qui était attendu par le public ? À cet égard, la maison d'édition L'Association, en France, qui, à ses débuts du moins, regroupait les forces vives de l'avant-garde, se donnait pour mandat d'offrir au public non pas des œuvres qui vendraient, mais des œuvres qui dureraient. Admettons qu'il y a, au départ, une résistance face à la production pour la masse. On résiste à quoi au juste ? À la facilité. À la recette. On ne remet pas en question le goût délicieux de la bande dessinée, on se demande s'il n'y aurait pas moyen de créer de nouvelles recettes avec des ingrédients tels que la 
case (ou son absence), la couleur, etc. Peut-être que l'expérimentation est une question d'attitude. Les bédéistes qui s'engagent sur la voie de l'expérimentation, seraient comme des anarchistes qui planifient la révolution. Parce qu'on a toujours besoin de préparer des révolutions pour le jour où on en aura besoin.

Dans la plupart des articles qui proposent des analyses de BD, les commentaires portent sur l'histoire ou les thèmes. Les éléments graphiques viennent appuyer les observations. L'analyse, inconsciemment peut-être, privilégie le récit sur la représentation parce que les théories critiques mises à contribution proviennent de spécialistes de la littérature. Les théories critiques qui ont été développées pour les arts visuels, comme la peinture et la photographie, présentent la lacune inverse: on ne donne pas assez de place à l'intrigue. Aujourd'hui, cependant, je voudrais passer dans le camp adverse et ne parler que de l'aspect graphique. Mais comment, justement, parler du style graphique ? On ne parle plus de l'aspect visuel en référence $\mathrm{au}$ «beau ». Faut-il emprunter la voie de Kandinsky qui, dans son livre Point, ligne plan, décrit les effets subjectifs des traits et des masses de couleur sur le public?

Mes observations porteront essentiellement sur Journal, mais les autres œuvres seront évoquées au besoin. De toutes les œuvres de Julie Delporte, Journal est sans doute la plus achevée. Je dirais même qu'il s'agit du parachèvement des expérimentations précédentes. En effet, on dirait que le projet, avec Le Dernier Kilomètre 1 et 2, consistait à « reconnaître le terrain », comme lorsqu'on explore l'orée d'une forêt avant d'y pénétrer plus profondément. Le style et les techniques développent des effets «brouillons », comme s'il s'agissait d'un travail en cours. Dans Je suis un raton laveur, la page est propre, mais le crayon de bois entre dans la danse. Les couleurs créent des formes.

Oui, je pense que la raison initiale, c'est que je ne savais pas - et je ne sais toujours pas, en quelque sorte - dessiner. J'ai commencé à dessiner au crayon de bois parce que ça me permettait d'effacer et de reprendre plusieurs fois de suite. Et comme je me suis mise à laisser les traces de la fabrication de mes dessins parce que j'avais cette idée de présenter un vrai objet, pas une planche nettoyée, il était logique de ne pas encrer, de laisser tout au crayon, et de laisser les traces de crayon effacé. Avec le crayon de couleur, ce n'est pas pareil parce que je ne peux pas effacer... Mais j'aime vraiment cela. Dans la bande dessinée contemporaine, je suis influencée par des auteurs qui utilisent le crayon ou par exemple, la peinture. Des gens comme Dominique Goblet, Johanna Hellgren, Amanda 
Vahamaki, etc. (Julie Delporte, entrevue du 31 juillet 2013)

Dans Journal, on note que rien ne ressemble à la « ligne claire », expression avec laquelle on qualifie le style d'Hergé, le créateur de Tintin. Afin de trouver sa propre personnalité graphique, une bédéiste doit-elle d'abord prendre une certaine distance avec l'héritage d'Hergé ? La ligne claire représente une forme de manichéisme graphique. Il y a le blanc du papier, puis le trait noir. Il n'y a pas de variations pour passer de l'un à l'autre. Les histoires d'Hergé ne peuvent être que manichéennes. Le trait noir, à l'encre, sépare des zones, chasse le flou. Pourtant, n'est-ce pas dans les zones floues que se trouvent les nuances de la vie ? Hergé avait placé la barre très haut avec son style très précis, très technique. Les albums qu'il a produits, sont imitables, mais cela demande tout un travail de recherche et de nombreux croquis préparatoires. Après Hergé, il semblait que l'artiste n'avait plus droit à l'imperfection.

Les deux numéros du Dernier kilomètre ont été réalisés à la mine de plomb. Cela donne un effet de «brouillon ». Le crayon à mine, c'est un choix qui pardonne puisqu'on peut effacer le trait et recommencer. On retrouve ainsi des couches archéologiques, laissées par exprès. Les anciens traits restent visibles, comme le souvenir des anciens amis. Il y a du ruban gommé, dont il faudra reparler. Il y a des zones laissées blanches, des zones grises, des zones hachurées. Il y a des zones qui ont été partiellement effacées. Comme un graffiti sur un monument, on comprend que «I was here ». L'auteure insère dans ses dessins des indices de sa présence.

Quant au crayon de bois, la technique employée dans Je suis un raton laveur, c'est plus difficile d'effacer. Les couleurs forment des blocs plutôt que d'être mélangées. Comme il est difficile de mélanger les couleurs au crayon de bois, la palette de couleur a des limites. Le crayon de bois, encore plus que la mine de plomb, semble contribuer à une esthétique de l'imprécision. L'absence de trait net, à l'encre, semble encore plus frappante pour le public habitué aux techniques classiques. On dirait qu'on passe dans le domaine de l'art visuel. Pourtant, il y a une histoire.

Pour le ruban de cassette, j'expérimentais le collage. Mais pour ce qui est du ruban adhésif, c'est une autre démarche. Je le mets en valeur sur mes dessins, comme toutes les autres traces de fabrication. En général, dans la bande dessinée, on efface ces traces de fabrication, on essaye de rendre les dessins propres. Au contraire, dans mon travail, je fais ressortir le sale et les traces de fabrication. Je 
travaille les fichiers sur Photoshop afin de mettre en valeur les traces de blanc correcteur, de tape, de papier. Quand je dessine sur du papier de carnet Molesquine qui est un peu jaune, je m'arrange pour laisser apparaître cette couleur jaune. Je crois que c'est encore cette idée de réaliser un objet qui semble vrai. Comme un document. Surtout avec Journal, qui est de toute façon un vrai Journal, donc un document, je laisse les ratures du document apparentes. Et puis il y a aussi un choix esthétique derrière cette démarche, le tape devient un matériel artistique, comme le crayon. Parfois dans mes dessins, j'en mets plus que je n'en ai besoin parce que je trouve cela beau. Je dessine avec du tape. (Julie Delporte, entrevue du 31 juillet 2013)

L'usage du tape évoque le collage, qui évoque le «scrap-book», une collection de documents et d'autres bricoles qui n'ont souvent d'intérêt que pour soi. Explorer cette collection c'est comme visiter un musée d'histoire. Les artefacts qu'on trouve dans l'aile égyptienne du Louvres NE SONT PAS l'Égypte des pharaons. Mais la collection d'objets permet d'IMAGINER ce qu'a pu être la vie à cet endroit et à cette époque. De même, Journal permet d'imaginer la vie de Julie Delporte.

L'un des traits de la bande dessinée, dont on a pu faire un critère définitoire pendant un certain temps, c'est la répétition d'éléments graphiques d'une case à l'autre. Rien de tel dans Journal. La constance, ici, c'est la variété. Il y a de la variété dans les techniques, comme on vient de le voir. Les dessins varient en qualité. Cela va du détaillé au naif. On dirait que le style varie avec l'humeur des jours. Buffon disait: «Le style, c'est l'homme [ou la femme, ajouterions-nous] ». Avec Julie Delporte, le style, c'est la vie de tous les jours.

La plupart des représentations de personnes et d'objets dans Journal ne sont pas exactement figuratives. Elles ne sont pas abstraites non plus. Ni primitives. Je voudrais qualifier les représentations «d'évocatrices ». Julie Delporte évoque la personne ou l'objet, mais sans tout montrer. Le public doit faire sa part et compléter l'évocation avec sa propre expérience au besoin. Ce qui expliquerait cette étrange impression de familiarité, de «déjà-vécu » que l'on ressent devant certains dessins.

Pourrait-on dire que les choix faits par Julie Delporte renforcent sa présence dans son œuvre ? Dans Journal, on peut noter (ô l'évidence !) qu'il s'agit d'un journal, écrit par soi et destiné à soi. Le public se complaît-il alors dans le voyeurisme ? Je crois plutôt que l'auteure 
lance une invitation. Mais je m'éloigne d'une analyse qui se veut purement graphique. Je veux seulement ajouter que le journal offre une discontinuité narrative dans une continuité chronologique, puisque chaque jour apporte une anecdote ou une réflexion au projet. C'est une collection d'événements.

Il y a des animaux, il y a des humains et il y a aussi des animaux anthropomorphisés, en particulier dans les deux numéros de Dernier Kilomètre. Dans les fables et les récits pour enfants, le procédé d'anthropomorphisme permet de rendre une situation universelle ou de créer une distance sécuritaire. L'anthropomorphisme chez Julie Delporte semble avoir d'autres fonctions. Il y a peut-être le besoin de rendre les personnages anonymes (une manière de se protéger du danger, comme Julie Delporte l'explique dans son essai Bédé-réalité), mais il y a aussi un amalgame. Si j'aime mes enfants et que j'aime les oiseaux, pourquoi ne pas représenter mes enfants sous forme d'oiseaux ? L'amalgame serait une autre forme de collection.

\section{Bibliographie}

Delporte, Julie. Encore ça. 2008.

--. Le Dernier Kilomètre No. 1, 2011.

- -. Le Dernier Kilomètre No. 2, 2012.

- -. La Bédé-Réalité. Colosse.

--. Journal. Toronto : Koyama P, 2013

--. Je suis un raton laveur. Montréal : La Courte Échelle, 2013.

Kandinsky, Wassily. Point ligne plan. Paris : Gallimard, 1991.

McCloud, Scott. Understanding Comics. New York : Harper Collins, 1994 (1993). 